\title{
Comorbilidad de TEA y TDAH: revisión sistemática de los avances en investigación
}

\author{
Jennifer Rico-Moreno ${ }^{1 *}$ y Raúl Tárraga-Mínguez ${ }^{2}$ \\ ${ }^{1}$ Generalitat de Catalunya. Departamento de Educación. Barcelona (España). \\ ${ }^{2}$ Departamento de Didáctica y Organización Escolar. Universidad de V alencia. Valencia (España).
}

\begin{abstract}
Resumen: La reciente publicación del DSM-5, introduce la novedad de aceptar la posibilidad de un diagnóstico comórbido de los Trastorno de Espectro Autista (TEA) y el Trastorno por Déficit de Atención/Hiperactividad (TDAH).

En este artículo se realiza una revisión de trabajos que han estudiado esta comorbilidad. Se revisaron un total de 28 investigaciones, estableciendo 4 temáticas diferentes. La investigación muestra tasas variables de comorbilidad de TEA y TDAH dependiendo de la edad y procedencia de las muestras, y de los criterios diagnósticos empleados. Cuando se da la comorbilidad de TEA y TDAH aumenta la severidad de la sintomatología autista y de trastornos externalizantes e internalizantes. Sin embargo, ello no parece relacionarse con un empeoramiento del estrés parental ni de la calidad de vida familiar.

Palabras clave: Trastorno de Espectro Autista; Trastorno por Déficit de Atención con Hiperactividad; comorbilidad; DSM-5; educación.
\end{abstract}

\section{Introducción}

En Mayo de 2013 se publicó el nuevo Manual Diagnóstico y Estadístico de los Trastornos Mentales (DSM-5), APA (2013). Esta nueva publicación introduce cambios que afectan, entre otros, al criterio diagnóstico de los Trastornos del Espectro Autista (TEA) y el Trastorno por Déficit de Atención con Hiperactividad (TDAH). Uno de los cambios más significativos y que finalmente ha sido tenido en cuenta en esta nueva versión es la eliminación del criterio de exclusión que impedía la posibilidad de diagnóstico comórbido de los TEA y TDAH. El presente trabajo constituye una revisión de los principales trabajos que, antes de la publicación del DSM-5, ya contemplaban la comorbilidad de TEA y TDAH. Esta revisión pretende tratar de ayudar a comprender e interpretar mejor los motivos que han llevado los autores del DSM a admitir la presencia comórbida de ambos trastornos en un mismo sujeto.

\section{Definición, prevalencia y diagnóstico del TEA y TDAH}

El autismo es un trastorno del neurodesarrollo caracterizado por déficits en la comunicación, la interacción social y el lenguaje, y por la presencia de intereses restringidos y comportamientos estereotipados, cuyos síntomas pueden identificarse en edades muy tempranas (Gargaro, Rinehart, Bradshaw, Tonge y Sheppard, 2011; Wing, Gould y Gillberg, 2011). Se estima que este trastorno afecta al $1 \%$ de la población (Baio, 2012; Baron-Cohen et al., 2009), afectando más al género masculino que al femenino en una proporción 4:1

* Dirección para correspondencia [Correspondence address]: Jennifer Rico-Moreno. Generalitat de Catalunya. Departamento de Educación. C/ Costa Rica, 26 CP: 08027. Barcelona (España).

E-mail: jrico23@xtec.cat
Title: ASD and ADHD comorbidity: a systematic review of advances in research.

Abstract: The recent publication of DSM-5, introduces as novelty to accept the comorbidity between the Autism Spectrum Disorder (ASD) and the Attention Deficit/Hyperactivity Disorder (ADHD).

This article reviews previous researches that have studied this comorbidity. A total of 28 investigations were reviewed, establishing 4 different themes. The research shows variable ASD and ADHD comorbidity rates depending on the age and origin of the samples, as well as the diagnostic criteria used. When ASD and ADHD comorbidity occurs, the autistic symptomatology and the externalizing and internalizing disorders increase. However, this does not seem to have a relation with a worsening of the parental stress or the quality of family life.

Key words: Autism Spectrum Disorders; Attention Deficit Hyperactivity Disorder; comorbidity; DSM-5; education.

(Fombonne, 2003). Durante las últimas décadas se ha observado un aumento considerable de la prevalencia de este trastorno (Lin et al., 2011; Matson y Kozlowski, 2011). Este aumento de la prevalencia puede estar explicado entre otros motivos, por los cambios en los conceptos y criterios de diagnóstico, los diversos métodos utilizados en los estudios, o la mayor disponibilidad de los servicios profesionales que contribuyen a una mejor identificación (Fombonne, 2005; Hsu, Chiang, Lin y Lin, 2012).

El TDAH es también un trastorno del neurodesarrollo caracterizado en este caso por las dificultades que experimentan los sujetos con la inatención, la hiperactividad y la impulsividad, las cuales interfieren en su funcionamiento diario. Diversas investigaciones han mostrado que no se trata de un trastorno que solo afecte en la niñez y la adolescencia, sino que frecuentemente muchos de sus síntomas siguen persistiendo durante la edad adulta (De Zwaan et al., 2012; Simon, Czobor y Bitter, 2013). La prevalencia del trastorno es dispar en función de las características de la población estudiada y de la metodología y el criterio diagnóstico empleado (Cardo et al., 2011). La revisión sistemática llevada a cabo por Polanczyk, de Lima, Horta, Biederman y Rohde (2007), en la que se contemplaban estudios de prevalencia de todo el mundo, mostró una prevalencia media del 5.29\%. Se ha observado una mayor prevalencia en el sexo masculino que en el femenino en una proporción 3:1 (Getahun et al., 2013) así como un aumento de la prevalencia del TDAH durante los últimos años (Getahun et al., 2013; Russell, Rodgers, Ukoumunne y Ford, 2014).

\section{Implicaciones del DSM-5 en el diagnóstico del TEA y TDAH}

La publicación del nuevo manual DSM-5 de la APA ha incluido diversos cambios que afectan directamente al diagnóstico del TEA y del TDAH. En cuanto a los TEA, se 
eliminan las categorías diagnósticas que incluían los "Trastornos Generalizados del Desarrollo", y pasan a estar incluidas dentro de una única categoría "Trastorno de Espectro Autista", en la que se establecerá el nivel de afectación del sujeto gracias a un baremo de severidad de los síntomas y de afectación. Existe cierta controversia respecto a este cambio. Mientras algunos estudios como los de Kaland (2011) y Ritvo (2012) sobre la fiabilidad de este nuevo criterio diagnóstico apuntan a que este no es muy sensible y puede afectar en la recepción de servicios sociales de aquellos niños que queden excluidos del diagnóstico, resultados de investigaciones como las de Frazier et al. (2012) o Huerta, Bishop, Duncan, Hus y Lord (2012) aportan datos que demuestran que este nuevo criterio tiene una mayor especifidad.

Los criterios diagnósticos del TDAH también han sido objeto de cambio. La nueva versión del DSM incluye este trastorno dentro de los "Trastornos del Neurodesarrollo" y amplía la edad de diagnóstico, teniendo en cuenta la posibilidad de que sus síntomas sigan presentes en la edad adulta (Martín-Fernández, 2013). Sin duda, uno de los cambios más relevantes es la aceptación de la posibilidad de comorbilidad de este trastorno con los TEA. Este nuevo criterio diagnóstico tendrá implicaciones interesantes en la práctica, abriéndose un amplio abanico de opciones terapéuticas que podrán ser utilizadas con los sujetos con diagnóstico comórbido de TEA y TDAH. A partir de este momento se contemplará en conjunto toda la sintomatología (sin centrarse tan solo en un subgrupo de síntomas), lo que permitirá dar respuesta a todas las necesidades que presenten estos sujetos.

Debido a la reciente aceptación de esta comorbilidad y la escasa disponibilidad de estudios sobre la misma (De la Iglesia y Olivar, 2012) nos proponemos en el presente trabajo, realizar una revisión de los trabajos recientes que han puesto de manifiesto la relación entre estos dos trastornos y sus consecuencias educativas. Esta revisión nos permitirá analizar además las causas que han llevado finalmente a la APA a aceptar esta comorbilidad.

\section{Material y métodos}

\section{Procedimiento}

En primer lugar, se realizó una búsqueda de las unidades de análisis con las que se realizaría la revisión de artículos. Para ello, introdujimos las palabras clave: $A D H D, A S D$, comorbidity, academic functioning, parental stress, education, prevalence, DSM-5, social abilities, quality of life y disruptive behavior (tanto en español como en inglés) en las bases de datos ERIC y Psic Info. Además, se rastreó la bibliografía de aquellos artículos que más directamente trataban el tema de la comorbilidad de TEA y TDAH, con el fin de localizar nuevos artículos no devueltos por las bases de datos en las búsquedas.

De los resultados obtenidos en las diferentes búsquedas se descartaron aquellos que no cumplieran alguno de los siguientes criterios:
Presentar resultados de un trabajo empírico.

- Incluir a un grupo de participantes con diagnóstico de TEA+TDAH durante las etapas de la infancia y adolescencia (menores de 18 años).

- Haber sido publicados entre los años 2004 y 2015.

- Estar redactados en español o inglés.

Tras establecer estos criterios, se obtuvieron un total de 28 artículos. Tras localizarlos físicamente, se procedió a su lectura, estableciéndose la siguiente clasificación según su contenido: 11 documentos que analizan la prevalencia de la comorbilidad entre TEA y TDAH, 12 documentos que analizan la severidad de la sintomatología en los sujetos con TEA+TDAH, 2 documentos que analizan el rendimiento escolar en los sujetos con TEA+TDAH y 4 documentos que analizan la calidad de vida de los sujetos con TEA+TDAH y sus familias.

\section{Resultados}

Los resultados de la revisión de documentos llevada a cabo en este estudio se organizan en diferentes secciones que tienen su correspondencia con los tópicos identificados dentro del área de estudio.

\section{Comorbilidad entre TEA y TDAH}

Pese a que las versiones anteriores al DSM-5 no contemplaban la posibilidad de emitir un diagnóstico comórbido de TEA y TDAH, la literatura científica ya contemplaba y estudiaba esta comorbilidad en varias investigaciones.

Los estudios de prevalencia sobre la comorbilidad de TEA y TDAH se han abordado mediante dos metodologías: en algunos casos se han recuperado datos de grandes estudios epidemiológicos ya realizados, que inicialmente se centraban solo en uno de los dos trastornos, pero en los que se evaluaba sintomatología del otro, lo que permite establecer la prevalencia de la comorbilidad de ambos (Hanson et al., 2013; Jang, et al., 2013a; Reiersen, Constantino, Volk y Todd, 2007; Russell et al., 2014; Simonoff et al., 2008; Turygin, Matson y Tureck, 2013); mientras que en otros casos, se trata de estudios con muestras más modestas cuyo foco estaba directamente puesto desde el inicio en evaluar la presencia comórbida de TEA y TDAH (Gjevik, Eldevik, Fjæran-Granum y Sponheim, 2011; Grzadzinski et al., 2011; Lee y Ousley, 2006; Mannion, Leader y Healy, 2013; Sinzig, Walter y Doepfner, 2009).

Los resultados de los estudios que han analizado la incidencia de la comorbilidad de TEA y TDAH se sintetizan en las Tablas 1 y 2 , en las que se incluyen los estudios que han analizado la prevalencia de la comorbilidad de TEA y TDAH, separados en función de si evalúan la presencia de TDAH en muestras de niños con TEA, o si por el contrario el enfoque escogido es el de evaluar la presencia de TEA en muestras de niños con TDAH. 
Tabla 1. Estudios que analizan la presencia de TDAH en estudiantes con TEA.

\begin{tabular}{llll}
\hline Autor (año) & N, Edad: media (desviación típica) & Instrumentos/criterios diagnóstico & Resultados \\
\hline Gjevik et al. & N=71 (58 niños, 13 niñas). & TEA: ADI-R. & Comorbilidad TEA+TDAH: 31\% (n= \\
(2011) & Edad: 11.8 años (3.3). & TDAH: criterios DSM-IV. & 22). De ellos: TDAH-I: $\mathrm{n}=15(68.18 \%) ;$ \\
& $\begin{array}{l}\text { Procedencia muestra: estudiantes escolari- } \\
\text { zados en un centro especializado en TEA } \\
\end{array}$ & & TDAH-HI: $\mathrm{n}=4(18.18 \%) ;$ TDAH-C: \\
& o que recibían apoyo puntual de dicho & &
\end{tabular}

Hanson et $\quad \mathrm{N}=1.838$ (1.637 niños, 201 niñas). De al. (2013) ellos: $1.631(88.7 \%)$ con TA, $157(8.5 \%)$ con TGD-NE y $50(2.7 \%)$ con SA. Edad: 8.93 (3.49).

Procedencia muestra: submuestra del $\mathrm{Si}$ mons Simplex Collection (EEUU).

Jang et al. $\quad \mathrm{N}=346$ (251 niños, 95 niñas) con TEA y 1851 niños con desarrollo atípico, pero sin TEA. Se controló la procedencia étnica de los participantes.

Edad grupo TEA: 25.89 meses (4.82).

Rango: 12-39 meses

Procedencia muestra: submuestra del estudio sobre intervención temprana Early Steps (EEUU).

Lee y Ousley $\quad \mathrm{N}=83$ (66 niños, 17 niñas). De ellos: 58 (2006) $\quad(69.88 \%)$ con TA, $12(14.46 \%)$ con SA y $13(15.66 \%)$ con TGD-NE.

Edad: 11.2 (3.9).

Procedencia muestra: niños y adolescentes derivados a un programa universitario de intervención psicofarmacológica (EEUU).

Mannion et $\mathrm{N}=89$ (74 niños, 15 niñas).

al. (2013) Edad: 9 años (39.53 meses).

Procedencia muestra: colegios, servicios especializados en TEA, grupos de apoyo a padres y foros online (Irlanda).

Russell et al. $\mathrm{N}=86$ (72 niños, 14 niñas) con diagnósti(2014) co de TEA.

Edad: 7.2 (0.3).

Procedencia muestra: estudio epidemiológico Millennium Cohort Study (Inglaterra).

Simonoff et $\mathrm{N}=112$ (98 niños, 14 niñas).

al. (2008) Edad: 11.5 años (rango 10-13.9).

Procedencia muestra: Special Needs and Autism Project (Inglaterra).

Sinzig et al. $\quad \mathrm{N}=83$ (70 niños, 13 niñas). De ellos: 9 (2009) (10.84\%) con TA, 30 (36.14\%) con AAF y $44(53.01 \%)$ con SA.

Edad: 11.7 (3.3).

Procedencia muestra: Alemania.
TEA: ADOS y ADI-R; criterios DSM-IV para TGD. TDAH: CBCL, TRF.

TEA: MCHAT; BISCUIT-Part 2; criterios DSM-5.

Sintomatología TDAH: subescala de inatención/impulsividad del BISCUIT-Part 2.

TEA: entrevista semiestructurada basada en criterios DSM-IV; Autism Behavior Checklist, GARS.

TDAH: ADHD Rating Scale (basada en criterios DSM-IV).

TEA: criterios DSM-IV.

TDAH: ASD-Comorbid for Children.

Existencia de un diagnóstico de TEA y/o TDAH por parte de un profesional médico o del ámbito de la salud.

TEA: ADI-R y ADOS. Criterios para autismo de ICD-10.

TDAH: criterios DSM-IV.

TEA: Diagnostic Checklist for PDD; PDD Rating Scale for Teachers and Parents (basados en criterios DSM-IV); ADI-R y ADOS.

TDAH: Diagnostic Checklist for ADHD; ADHD Rating Scale for

Teachers and Parents (basados en criterios de DSM-IV e ICD-10).
Comorbilidad TEA+TDAH: entre un $2.6 \%(n=21)$ y un $17.6 \%(n=324)$, en función de los instrumentos disponibles en la muestra.

Comorbilidad TEA+TDAH: los niños con TEA presentaban niveles de inatención e impulsividad significativamente mayores que los niños con desarrollo atípico sin TEA. Los niños afroamericanos presentaban mayores niveles de comorbilidad que los niños de otras procedencias étnicas.

Comorbilidad TEA+TDAH: 78\% $(\mathrm{n}=65)$. De ellos: TDAH-I: $\mathrm{n}=19$ (29.23\%); TDAH-HI: $\mathrm{n}=5$ (7.69\%); TDAH-C: $\mathrm{n}=41(63.08 \%)$.

El grupo con TA presentaba una mayor prevalencia de TDAH-HI en comparación a los otros subgrupos de TEA.

Comorbilidad TEA+TDAH: 18\% $(\mathrm{n}=16)$.

De ellos, un $87.5 \%(n=14)$ eran niños.

Comorbilidad TEA+TDAH: $9.3 \%(\mathrm{n}=$ 8).

Comorbilidad TEA+TDAH: $28.2 \%$.

Comorbilidad TEA+TDAH: $53 \%$

$(n=44)$. De ellos:

TDAH-I: $\mathrm{n}=20$ (45.5\%);

TDAH-HI: $\mathrm{n}=10(22.73 \%)$;

TDAH-C: $\mathrm{n}=14(31.82 \%)$.
Turygin et $\mathrm{N}=656$ (486 niños, 170 niñas). al. (2013)
Edad: menores de 36 meses.
TEA: Criterios para TEA de DSM- Comorbilidad TEA+TDAH: 4.65\%. No 5. hubo diferencias respecto a género o 


\begin{tabular}{|c|c|c|c|}
\hline Autor (año) & N, Edad: media (desviación típica) & Instrumentos/criterios diagnóstico & Resultados \\
\hline & $\begin{array}{l}\text { Procedencia: programa de intervención } \\
\text { temprana a nivel estatal (EEUU). }\end{array}$ & $\begin{array}{l}\text { TDAH: BISCUIT Part } 2 \text { (basado en } \\
\text { criterios DSM-IV). }\end{array}$ & subtipo de TDAH. \\
\hline \multicolumn{4}{|c|}{$\begin{array}{l}\text { Nota: ADI-R: Autism Diagnostic Interview; TDAH-I: TDAH predominantemente inatento; TDAH-HI: TDAH predominantemente Hiperactivo-Impulsivo; } \\
\text { TDAH-C: TDAH combinado; TA: Trastorno Autista; TGD-NE: Trastorno Generalizado del Desarrollo-No especificado; SA: Síndrome de Asperger; } \\
\text { ADOS: Autism Diagnostic Observation Schedule; TGD: Trastorno Generalizado del Desarrollo; CBCL: Child Behavior Checlits; TRF: Teacher Report Form; MCHAT: } \\
\text { Modified Checklist for Autism in Toddlers BISCUIT: Baby and Infant Screen for Children with Autism Traits; GARS: Gilliam Autism Rating Scale; ICD-10: International Sta- } \\
\text { tistical Classification of Diseases and Related Health Problems; AAF: Autismo de Alto Funcionamiento; PDD: Pervasive Developmental Disorders. }\end{array}$} \\
\hline
\end{tabular}

Tabla 2. Estudios que analizan la presencia de TEA en estudiantes con TDAH.

\begin{tabular}{|c|c|c|c|}
\hline Autor (año) & $N$, Edad: media (desviación típica) & Instrumentos & Resultados \\
\hline $\begin{array}{l}\text { Grzadzinski } \\
\text { et al. (2011) }\end{array}$ & $\begin{array}{l}\text { N= } 75 \text { (60 niños, } 15 \text { niñas). De ellos: } 38(50.67 \%) \\
\text { con TDAH-C, } 27 \text { (36\%) con TDAH-I., } 6(8 \%) \\
\text { con TDAH-HI y } 4(5.33 \%) \text { con TDAH-NE. } \\
\text { Edad: entre } 7.1 \text { y } 17.8 \text { años. } \\
\text { Procedencia muestra: los participantes fueron re- } \\
\text { clutados a través de anuncios en prensa, flyers y } \\
\text { desde grupos de apoyo a padres (EEUU). }\end{array}$ & $\begin{array}{l}\text { TDAH: Conners' Parent Rating } \\
\text { Scale; Schedule of Affective Disorders } \\
\text { and Schizophrenia for Children. } \\
\text { TEA: SRS (basado en criterios } \\
\text { DSM-IV); Children's Communica- } \\
\text { tion Checklist-2. }\end{array}$ & $\begin{array}{l}\text { Comorbilidad TEA+TDAH: entre } \\
1 / 3 \text { y } 1 / 5 \text { de los niños con TDAH } \\
\text { presentaban elevados niveles de sin- } \\
\text { tomatología autista (en función del } \\
\text { instrumento utilizado). }\end{array}$ \\
\hline $\begin{array}{l}\text { Reiersen et } \\
\text { al. (2007) }\end{array}$ & $\begin{array}{l}\mathrm{N}=134 \text { (115 niños, } 19 \text { niñas). De ellos: } 45 \\
(33.58 \%) \text { con TDAH-C, } 75(55.97 \%) \text { con TDAH- } \\
\text { I y } 14(10.45 \%) \text { con TDAH-HI. } \\
\text { Edad: } 12.4 \text { (DT= } 3.0) \text {. } \\
\text { Procedencia muestra: participantes provenientes } \\
\text { de un estudio epidemiológico con gemelos } \\
\text { (EEUU). }\end{array}$ & $\begin{array}{l}\text { TDAH: Missouri Assessment of } \\
\text { Genetics Interview for Children (ba- } \\
\text { sada en criterios DSM-IV). } \\
\text { TEA: SRS. }\end{array}$ & $\begin{array}{l}\text { Comorbilidad TEA+TDAH: } \\
38.86 \% \text {. En función de subtipo } \\
\text { TDAH y sexo: } \\
\text { TDAH-HI: } 0 \% \text {. } \\
\text { TDAH-I: } 14 \% \text { chicos; } 33 \% \text { chicas. } \\
\text { TDAH-C: } 32 \% \text { chicos; } 75 \% \text { chicas. }\end{array}$ \\
\hline
\end{tabular}

Nota: TDAH-C: TDAH combinado; TDAH-I: TDAH predominantemente Inatento; TDAH-HI: TDAH predominantemente Hiperactivo-Impulsivo; TDAH-NE: TDAH-No Especificado; SRS: Social Responsiveness Scale.

\section{Severidad de las conductas autistas y de los compor- tamientos disruptivos}

Por otra parte, diversos estudios se han propuesto estudiar si la comorbilidad de TEA y TDAH tiene un efecto en la severidad de las conductas autistas (Gadow, DeVincent y Pomeroy, 2006; Goldstein y Schwebach, 2004; Rao y Landa, 2014; Sprenger et al., 2013; Yerys et al., 2009), en la severidad de los comportamientos disruptivos (Holtmann, Bölte y Pustka, 2007; Goldin, Matson, Tureck, Cervantes y Jang, 2013; Grzadzinski et al., 2011; Jang, Matson, Cervantes y
Goldin, 2013b; Konst, Matson y Turygin, 2013; Tureck, Matson, May y Turygin, 2013; Yerys et al., 2009), o en la sintomatología depresiva y otros trastornos internalizantes (Grzadzinski et al., 2011; Holtmann et al., 2007; Kotte et al., 2013). El enfoque que han adoptado la mayoría de estos trabajos es el de la comparación de la severidad de la sintomatología en al menos 2 grupos: un grupo de sujetos con TEA+TDAH y uno o dos grupos de sujetos que presentaban solo uno de estos diagnósticos de manera aislada.

La Tabla 3 ofrece una síntesis de los resultados de estos estudios.

Tabla 3. Severidad de las conductas autistas y de los comportamientos disruptivos.

\begin{tabular}{|c|c|c|c|}
\hline Autor (año) & N, Edad: media (desviación típica) & Instrumentos & Resultados \\
\hline $\begin{array}{l}\text { Gadow et al. } \\
(2006)\end{array}$ & $\begin{array}{l}\text { N= } 483 \text { ( } 398 \text { chicos, } 85 \text { chicas) en dos grupos de } \\
\text { edad: } \\
\text { 3-5 años: media }=4.2(0.8) \text {. } \\
\text { 6- } 12 \text { años: media }=8.3(1.9) \text {. } \\
\text { De ellos: } 170 \text { con TA, } 104 \text { con SA y } 209 \text { con } \\
\text { TGD-NE. }\end{array}$ & $\begin{array}{l}\text { ECI-4; CSI-4; } \\
\text { CBCL; TRF; } \\
\text { Conners Teacher. }\end{array}$ & $\begin{array}{l}\text { El grupo TEA+TDAH subtipo combinado pre- } \\
\text { sentó mayores niveles de sintomatología autista } \\
\text { de acuerdo a la valoración de los padres y mayo- } \\
\text { res problemas externalizantes e internalizantes }\end{array}$ \\
\hline $\begin{array}{l}\text { Goldin et al. } \\
\text { (2013) }\end{array}$ & $\begin{array}{l}\text { Grupo TEA+TDAH: } n=47 \text { ( } 40 \text { niños, } 7 \text { niñas). } \\
\text { Edad: } 9.60 \text { ( } 3.3 \text { ). } \\
\text { Grupo TEA: } n=255 \text { ( } 206 \text { niños, } 49 \text { niñas). Edad: } \\
7.88 \text { (3.5). }\end{array}$ & $\begin{array}{l}\text { ASD-Comorbid- } \\
\text { Child-Version. }\end{array}$ & $\begin{array}{l}\text { El grupo TEA+TDAH presentó mayores nive- } \\
\text { les de rabietas que el grupo TEA y que un grupo } \\
\text { TDAH. }\end{array}$ \\
\hline
\end{tabular}




\begin{tabular}{|c|c|c|c|}
\hline Autor (año) & $N$, Edad: media (desviación típica) & Instrumentos & Resultados \\
\hline $\begin{array}{l}\text { Goldstein } \quad y \\
\text { Schwebach } \\
(2004)\end{array}$ & $\begin{array}{l}\text { Grupo TEA+TDAH: } \mathrm{n}=6 . \\
\text { Grupo TEA: } \mathrm{n}=6 . \\
\text { Edad: } 8.5(3.6)\end{array}$ & $\begin{array}{l}\text { Conners Rating Sca- } \\
\text { le (versiones de } \\
\text { padres y profeso- } \\
\text { res). }\end{array}$ & $\begin{array}{l}\text { El grupo TEA+TDAH presentaba mayores difi- } \\
\text { cultades en actividades de la vida diaria que el } \\
\text { grupo TEA, de acuerdo a valoración de padres y } \\
\text { profesores. }\end{array}$ \\
\hline $\begin{array}{l}\text { Grzadzinski et } \\
\text { al. (2011) }\end{array}$ & $\begin{array}{l}\mathrm{N}=75 \text { (60 niños, } 15 \text { niñas). } \\
\text { Descrita en tabla } 2 .\end{array}$ & $\begin{array}{l}\text { SRS, Children's } \\
\text { Communication } \\
\text { Checklist-2. }\end{array}$ & $\begin{array}{l}\text { El grupo TEA+TDAH presentó mayores nive- } \\
\text { les de conducta oposicionista y mayores puntua- } \\
\text { ciones de depresión que el grupo TDAH. }\end{array}$ \\
\hline
\end{tabular}

Holtmann et Grupo TGD+TDAH: $\mathrm{n}=89$ (65 chicos, 24 chi- CBCL.

al. (2007) cas). Edad: 10.7 (4.7).

Grupo TGD: $n=93$ (76 chicos, 17 chicas). Edad: $10.4(4.7)$

El grupo TEA+TDAH presentó mayores niveles de problemas internalizantes y externalizantes que el grupo TEA. Las mayores diferencias se dieron en el área de interacción social.

Jang et al. Grupo TEA+TDAH: $\mathrm{n}=38$ (35 niños, 6 niñas).

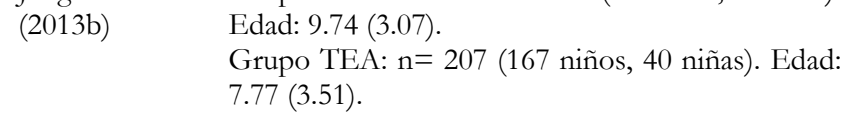
7.77 (3.51).

ASD-Comorbid-

Child-Version.

Konst et al. $\mathrm{N}=347$ (273 niños, 74 niñas). Edad: 8.51 (3.67).

(2013) Grupo TEA+TDAH: $n=49$.

Grupo TEA: $n=256$.

ASD-Comorbid-

Child-Version.

El grupo TEA+TDAH presentó mayores niveles de rabietas y problem as de conducta que el grupo TEA y que un grupo TDAH.

Kotte et al. Grupo TEA+TDAH: $\mathrm{n}=44$ (26 niños, 18 niñas). (2013) Edad: 9.8 (2.7) Grupo TDAH: $\mathrm{n}=198$ (107 niños, 91 niñas). Edad: 11.0 (3.14). SAICA; Moos más severas de ajuste escolar y familiar, así como Family Environ- un mayor grado de problemas internalizantes y ment Scale.

Rao y Landa Grupo TEA+TDAH: $n=18$ (18 niños). Edad: (2014) $\quad 5.67(1.4)$. Grupo TEA: $n=44$ (33 niños, 11 niñas). Edad: tive Behavior Scale. 5.59 (1.2).

BASC-2; SRS; El grupo TEA+TDAH presentó mayores difiVineland Adapta- cultades en el funcionamiento adaptativo y so-

Sprenger et al. Grupo TEA+TDAH: $\mathrm{n}=56$ (50 niños, 6 niñas). ADOS, ADI-R, (2013) De ellos, 18 AAF, 7 TA y 31 SA. Edad: 12.68 SRS, CBCL. (4.3). Grupo TEA. N= 70 (67 niños, 3 niñas). De ellos, 14 AAF, 10 TA y 46 SA. Edad: 15.1 (4.7).

Tureck et al. Grupo TEA+TDAH n=42 (36 niños, 6 niñas). (2013)

\section{Edad: 9.50 (3.13).} Grupo TDAH n=43 (26 niños, 17 niñas). Edad: 9.49 (3.13).

ASD-ComorbidChild-Version; ASD Bebavior Problems for Children.

Yeris et al. Grupo TEA+TDAH: $\mathrm{n}=21$ (18 niños, 3 niñas). SRS, BASC. (2009) De ellos: $9(42.86 \%)$ con TA, $6(28.58 \%)$ con SA y $6(28.58 \%)$ con TGD-NE. Edad: 9.65 (1.62). Grupo TEA: $\mathrm{n}=28$ (20 niños, 8 niñas). De ellos: $13(46.43 \%)$ con TA, $11(39.29 \%)$ con SA y 4 (14.29\%) con TGD-NE. Edad: 9.7 (2.12). externalizantes que el grupo TDAH. cial, así como más movimientos estereotipados que el grupo TEA.

El grupo TEA+TDAH presentó mayores niveles de rabietas que el grupo TEA y que un grupo TDAH.

El grupo TEA+TDAH presentaba mayores niveles de sintomatología autista que el grupo TEA de acuerdo a puntuaciones en ADI-R y SRS.

El grupo TEA+TDAH presentó mayores niveles de rabietas y conductas externalizantes que el grupo TDAH.

El grupo TEA+TDAH presentó mayores niveles de sintomatología autista que el grupo TEA de acuerdo a puntuaciones SRS, así como mayores puntuaciones en problemas externalizantes, atencionales y de hiperactividad de acuerdo a puntuaciones en BASC.

Nota: TA: Trastorno Autista; SA: Síndrome de Aspeger; TGD-NE: Trastorno Generalizado del Desarrollo-No especificado; ECI-4: Early Cbildbood Inventory-4; CSI-4: Child Symptom Inventory-4; CBCL: Check Behavior Checklist, TRF: Teacher Report Form; SRS: Social Responsiveness Scale; Kiddie: Kiddie Schedule for Affective Disorders and Schizopbrenia; SAICA: Social Adjustment Inventory for Children and Adolescents; BASC: Behavior Assessment System for Children; ADOS: Autism Diagnostic Observation Schedule; ADI-R: Autism Diagnostic Interview: AAF: autismo de alto funcionamiento.

\section{Rendimiento académico}

Aunque en menor medida que los tópicos anteriores, la investigación también ha prestado atención a la influencia que ejerce sobre el rendimiento académico la presencia co- mórbida de TEA y TDAH en comparación al rendimiento académico que se da cuando está presente solo uno de estos dos trastornos. La Tabla 4 resume los resultados de los trabajos de Barnard-Brak, Sulak y Fearon (2011) y May, Ri- 
nehart, Wilding y Cornish (2013), que han analizado este tó- $\quad$ pico.

Tabla 4. Rendimiento académico.

\begin{tabular}{|c|c|c|c|}
\hline Autor (año) & $N$ & Instrumentos & Resultados \\
\hline $\begin{array}{l}\text { Barnard-Brak } \\
\text { et al. (2011) }\end{array}$ & $\begin{array}{l}\mathrm{N}=2.844 \text { niños con TDAH (edades de } 6 \text { a } 12 \\
\text { años) a los que se realizó un seguimiento longi- } \\
\text { tudinal durante } 3 \text { años. } \\
\text { Evaluación 1: el } 10.20 \% \text { cumplía los criterios } \\
\text { diagnósticos de TEA. Evaluación } 2: \text { los cumplía } \\
\text { el } 12 \% \text {. Evaluación: los cumplía el } 16 \% \text {. }\end{array}$ & WJ-III-R & $\begin{array}{l}\text { Se evidenció una relación inversa entre la presencia de } \\
\text { trastornos comórbidos al TDAH (entre ellos el TEA) } \\
\text { y el rendimiento académico. }\end{array}$ \\
\hline $\begin{array}{l}\text { May et al. } \\
(2013)\end{array}$ & $\begin{array}{l}\mathrm{N}=64 \text { (32 niños, } 32 \text { niñas). Edad: } 9.9 \text { (1.85) } \\
\text { con TA o SA. }\end{array}$ & WIAT-II & $\begin{array}{l}\text { Se analizó el papel de la atención en el logro académi- } \\
\text { co de sujetos TEA. La capacidad de cambiar la aten- } \\
\text { ción jugaba un papel importante en el área de las ma- } \\
\text { temáticas. }\end{array}$ \\
\hline
\end{tabular}

Nota: WJ-III-R: Woodcock Johnson-III; TA: Trastorno Autista; SA: Síndrome de Asperger; WIAT-II: Weschler Individual Achievement Test-II.

Calidad de vida de los sujetos TEA+TDAH y sus familias

Por último, hasta la fecha, un único trabajo se ha interesado en analizar si la presencia comórbida de TEA y TDAH ejerce mayor impacto en la calidad de vida de los sujetos con este diagnóstico (Sikora, Vora, Coury y Rosenberg, 2012) y tres trabajos han analizado este mismo constructo de calidad de vida en las familias de los niños con TEA+TDAH (Miranda, Tárraga, Fernández, Colomer y Pastor, 2015; Van Steijn, Oerlemans, van Aken, Buitelaar y Rommelse, 2013, 2014). La Tabla 5 sintetiza los resultados de estos trabajos.

Tabla 5. Calidad de vida de los sujetos TEA+TDAH y sus familias.

\begin{tabular}{|c|c|c|c|}
\hline Autor (año) & $\mathrm{N}$ & Instrumentos & Resultados \\
\hline $\begin{array}{l}\text { Miranda et } \\
\text { al. (2015) }\end{array}$ & $\begin{array}{l}\text { Grupo TEA+TDAH: } n=21 \text { (20 niños, } 1 \text { niña). } \\
\text { Edad: } 5.82(1.09) \text {. } \\
\text { Grupo TEA: } n=23 \text { (20 niños, } 3 \text { niñas). Edad: } \\
6.23(1.10) .\end{array}$ & PSI & $\begin{array}{l}\text { No hubo diferencias entre ambos grupos en el indicador } \\
\text { global de estrés parental, pero el grupo TEA+TDAH pre- } \\
\text { sentó diferencias en las escalas de estrés parental relaciona- } \\
\text { do con la salud y el apoyo entre los padres como pareja. }\end{array}$ \\
\hline $\begin{array}{l}\text { Sikora et al. } \\
(2012)\end{array}$ & $\begin{array}{l}\text { N= } 3066 \text { (2586 niños, } 480 \text { niñas). } \\
\text { Edad: } 1737 \text { entre } 2 \text { y } 5 \text { años; } 1020 \text { entre } 6 \text { y } 11 \\
\text { años; } 309 \text { entre } 12 \text { y } 18 \text { años. De ellos, } 2041 \\
\text { con TA, } 273 \text { con SA y } 752 \text { con TGD-NE. } \\
\text { Grupo TEA+TDAH: } n=1267 . \\
\text { Grupo TEA: } n=1799 .\end{array}$ & $\begin{array}{l}\text { CBCL;Vinela } \\
\text { nd; PQoL. }\end{array}$ & $\begin{array}{l}\text { El grupo TEA+TDAH presentaba peores indicadores en } \\
\text { todas las escalas del cuestionario pediátrico de calidad de } \\
\text { vida. }\end{array}$ \\
\hline $\begin{array}{l}\text { Van Steijn } \\
\text { et al. } \\
(2013)\end{array}$ & $\begin{array}{l}\text { Grupo TEA+TDAH: } n=33 \text { (30 niños, } 3 \text { ni- } \\
\text { ñas). Edad: } 12.4 \text { (3.1). } \\
\text { Grupo TEA: } n=47 \text { ( } 39 \text { niños, } 8 \text { niñas). Edad: } \\
13.0(3.3) \text {. }\end{array}$ & $\begin{array}{l}\text { Parent-Child } \\
\text { Interaction } \\
\text { Questionnaire. }\end{array}$ & $\begin{array}{l}\text { En ambos grupos la sintomatología de TEA en los padres } \\
\text { (pero no en las madres) se relacionó con menos sentimien- } \\
\text { to de aceptación por parte del niño respecto a sus padres. } \\
\text { La sintomatología de TDAH en los padres (pero no en las } \\
\text { madres) se relacionó con menores habilidades de resolu- } \\
\text { ción de conflictos. }\end{array}$ \\
\hline $\begin{array}{l}\text { Van Steijn } \\
\text { et al. (2014) }\end{array}$ & $\begin{array}{l}\text { Grupo TEA+TDAH: } n=59 \text { (49 niños, } 10 \text { ni- } \\
\text { ñas). } \\
\text { Edad: } 10.9 \text { (3.3). } \\
\text { Grupo TEA: } n=48 \text { (41 niños, } 7 \text { niñas). Edad: } \\
12.0 \text { (4.2). }\end{array}$ & $\begin{array}{l}\text { PSI; Adult } \\
\text { Social Behavior } \\
\text { Questionnaire. }\end{array}$ & $\begin{array}{l}\text { No hubo diferencias entre los grupos TEA+TDAH y TEA } \\
\text { en estrés parental y sintomatología depresiva en los padres. }\end{array}$ \\
\hline
\end{tabular}

Nota: PSI: Parenting Stress Index; CBCL: Check Behavior Checklist; Vineland: Vineland Behavior Scales PQoL: Pediatric Quality of Life Inventory.

\section{Discusión}

El presente trabajo ofrece una revisión de investigaciones recientes que han estudiado la comorbilidad del TEA y TDAH. Consideramos que esta revisión puede contribuir a entender mejor los motivos que justifican la novedad que supone la aceptación de esta comorbilidad en el nuevo ma- nual de la APA: el DSM-5, ya que en la revisión llevada a cabo se pone de manifiesto que desde hace tiempo la literatura especializada ya venía dando cuenta de la presencia de esta comorbilidad en numerosos estudios.

Tras la revisión han emergido como aspectos más estudiados el interés por conocer la prevalencia de la coocurrencia de ambos trastornos y el estudio de si la presencia co- 
mórbida de ambos trastornos supone un aumento en la severidad de la sintomatología. En menor medida, también ha sido estudiada la influencia de la comorbilidad de TEA y TDAH en aspectos como el rendimiento académico y la calidad de vida de los sujetos diagnosticados y sus familias.

La prevalencia del diagnóstico comórbido TEA+TDAH se ha estimado en tasas muy dispares, que oscilan entre un 4.65\% (Turygin et al., 2013) y un 78\% (Lee y Ousley, 2006). Los motivos de esta disparidad de resultados radican en aspectos como la edad y procedencia de los participantes en los diferentes estudios, así como en los criterios y procedimientos empleados para el diagnóstico.

El estudio con las tasas de prevalencia más bajas, realizado por Turygin et al. (2013) se llevó a cabo en el seno de un programa de intervención temprana en el que los participantes tenían menos de 36 meses de edad, mientras que el resto de estudios de prevalencia (salvo el de Jang et al., 2013a) se han realizado con estudiantes de mayor edad.

La presencia de una tasa de prevalencia de TEA+TDAH tan baja (4.65\%) como la reportada por Turygin et al. (2013), parece sugerir que el desarrollo de los síntomas de TDAH no sigue el mismo ritmo en la población con y sin TEA, lo que tiene importantes repercusiones para la práctica educativa, ya que al no existir una presencia evidente de síntomas de inatención e impulsividad durante los primeros años de vida, probablemente las intervenciones tempranas no focalizarán en estos aspectos, pese a que el resto de investigaciones sobre este tópico parece indicar que en etapas posteriores del desarrollo, sí es muy probable que aparezcan estos síntomas.

Además, la diferente procedencia de las muestras empleadas en los estudios de prevalencia puede explicar también la disparidad de los resultados. En el estudio que halló la tasa de prevalencia de TEA+TDAH más alta, de hasta un 78\% (Lee y Ousley, 2006), los integrantes de la muestra participaban en un estudio de intervención psicofarmacológica, lo que supone un importante sesgo en la selección de la muestra empleada en el estudio que explica probablemente la elevada tasa de comorbilidad encontrada.

También la determinación de la persona que realiza el diagnóstico (los autores del estudio de investigación, o profesionales ajenos al mismo), influye en la prevalencia del diagnóstico de TEA+TDAH. En el estudio de Russell et al. (2014), el diagnóstico venía dado por profesionales de la salud ajenos al propio estudio. Estos profesionales deben guiarse por las recomendaciones de los sistemas de clasificación como el DSM, que en sus versiones anteriores no contemplaba la posibilidad de un diagnóstico comórbido de TEA y TDAH. Pese a ello, un $9.3 \%$ de los sujetos con diagnóstico de TEA del estudio de Russell et al. (2014) tenían también diagnóstico de TDAH, lo que pone de nuevo en evidencia que incluso antes de que el DSM admitiera esta comorbilidad, en la práctica clínica ya se contemplaba.

Por último, el procedimiento y los criterios empleados para realizar el diagnóstico, influyen también poderosamente en el resultado de prevalencia. De hecho, en el trabajo de
Hanson et al. (2013) se hallaron dos tasas de prevalencia de la comorbilidad TEA+TDAH: un $17.6 \%$ cuando la valoración de los síntomas fue realizada por los padres, y una prevalencia mucho más reducida, del $2.6 \%$ cuando se emplearon como criterio simultáneamente las puntuaciones padres y profesores.

Tan solo 4 estudios de este tópico han analizado la relación con el TEA de los diferentes subtipos de TDAH. En los estudios de Lee y Ousley (2006) y Reiersen et al. (2007) el subtipo de TDAH más presente en el grupo comórbido fue el de TDAH combinado. Sin embargo, en los restantes estudios que se han adentrado a describir el subtipo de TDAH (Gjevik et al., 2011; Sinzig et al., 2009), el subtipo de TDAH más común en los sujetos con diagnóstico de TEA+TDAH fue el subtipo TDAH predominantemente inatento, por lo que los resultados a este respecto no son determinantes.

La constatación de la presencia conjunta de TEA y TDAH en numerosos casos, ha abierto la puerta a preguntarse por los aspectos compartidos por TEA y TDAH en el plano clínico, neuropsicológico y de neuroimagen. A este respecto, Gargaro et al. (2011), evidenciaron que, si bien cuando ambos trastornos se presentan de manera "pura", aislados el uno del otro, las evidencias muestran claramente que se trata de dos trastornos nítidamente diferenciados, existen aspectos clínicos y neuropsicológicos compartidos por ambos trastornos cuando se presentan de manera comórbida.

En cuanto al ámbito de la severidad de la sintomatología, los resultados obtenidos en nuestra investigación indican que la presencia del TDAH en los sujetos diagnosticados con TEA aumenta significativamente la severidad de la propia sintomatología autista (Gadow et al., 2006; Rao y Landa, 2014; Sprenger et al., 2013; Yerys et al., 2009), la presencia de rabietas y problemas de conducta (Goldin et al., 2013; Grzadzinski et al., 2011; Jang et al., 2013b; Konst et al., 2013; Tureck et al., 2013; Yerys et al., 2009), e igualmente aumenta la severidad de la sintomatología depresiva y otros trastornos internalizantes (Grzadzinski et al., 2011; Kotte et al., 2013).

$\mathrm{El}$ conjunto de estos resultados parece indicar que existe un efecto aditivo de los síntomas de TEA y de TDAH que supone un aumento de las dificultades para los niños con diagnóstico comórbido. Este agravamiento de los síntomas tiene importantes repercusiones para la práctica clínica y educativa, y constata la conveniencia de evaluar la presencia de sintomatología de TDAH en los niños con diagnóstico de TEA, para poder planificar intervenciones eficaces que atiendan al conjunto de las dificultades presentadas por los estudiantes, y no solo en a una parte de ellas, existiendo la posibilidad de ir modificando las intervenciones a medida que se vaya obteniendo más información acerca de la comorbilidad de los distintos trastornos (Holtmann et al., 2007).

Además, este efecto aditivo puede (al menos en parte) ser el responsable del empeoramiento del rendimiento académico de estos niños. A este respecto, el trabajo de May et 
al. (2013) constató que la capacidad de cambiar la atención jugaba un papel importante en el área de las matemáticas, la cual cuando es deficiente se asocia a un detrimento de los resultados funcionales en este área curricular. Los investigadores además hacen hincapié en la importancia que tiene el uso de las matemáticas en las habilidades de la vida diaria, como por ejemplo la estimación, la cocina y la realización de presupuestos, lo cual puede tener impactos significativos en la conducta adaptativa. Por su parte, Barnard-Brak et al. (2011) realizaron un seguimiento longitudinal a un grupo de estudiantes con TDAH en tres momentos temporales, en el que analizaron qué trastornos comórbidos al TDAH se encontraban presentes en los tres momentos. Los resultados mostraron claramente una relación inversa entre el número de trastornos comórbidos al TDAH y el rendimiento académico. En el estudio se contemplaron hasta 12 posibles diagnósticos comórbidos, de los que el autismo fue el tercer diagnóstico más presente en los tres momentos temporales, solo por detrás de las dificultades específicas de aprendizaje y el trastorno de lenguaje. Sin embargo, el trabajo de Barnard-Brack et al. (2011) no ofrece el rendimiento académico del grupo TEA+TDAH de manera aislada, sino que se presentan los datos de rendimiento diferenciando entre grupo TDAH con comorbilidad (de cualquiera de los 12 diagnósticos contemplados) y sin comorbilidad, por lo que no es posible ofrecer un perfil claro del tipo de dificultades que presentan los sujetos con TEA+TDAH.

Finalmente, en lo que respecta a la calidad de vida de los sujetos con TEA+TDAH y sus familias, los resultados parecen indicar que cuando se compara al grupo comórbido con los grupos de diagnósticos aislados, no se producen diferencias significativas entre los grupos en estrés parental y calidad de vida familiar (Miranda et al., 2015; Van Steijn et al., 2013, 2014), aunque sí en la calidad de vida de los propios sujetos (Sikora et al., 2012).

La investigación sobre estrés parental ha evidenciado en numerosas ocasiones que las familias de niños con TEA y con TDAH experimentan mayores niveles de estrés parental que las familias de niños sin diagnóstico. Estos resultados se han presentado de manera sistemática en los metaanálisis llevados a cabo por Hayes y Watson (2013) y Theule, Wiener, Tannock y Jenkins (2013).

Dado que la severidad de la sintomatología autista se ha mostrado en numerosas ocasiones como un importante predictor del estrés parental (Ekas y Whitman, 2010; Lyons, Leon, Phelps y Dunleavy, 2010; Pozo y Sarriá, 2014), parecería posible pensar que el efecto aditivo de la sintomatología de TEA y TDAH repercutiría negativamente en los niveles de estrés parental. Sin embargo, los trabajos incluidos en esta revisión no permiten realizar de manera tajante esta afirmación, ya que no se han hallado diferencias en estrés parental (Miranda et al., 2015), ni en calidad de vida familiar en familias de niños con TEA+TDAH y en familias con estos trastornos de manera aislada (Van Steijn et al., 2013, 2014). El estrés parental está en función de los elementos estresores, pero también se ve afectado por otros factores, como las capacidades y recursos de las propias familias de hacer frente a este estrés, así como por la propia dinámica del funcionamiento familiar, por lo que es bastante posible que estos elementos estén influyendo en los resultados sobre estrés parental.

Sin embargo, cuando el foco de lo evaluado se centra en los sujetos con diagnóstico comórbido (y no en sus familias), sí surgen diferencias importantes entre los sujetos con TEA+TDAH y los sujetos con solo uno de estos trastornos. Sikora et al. (2012), encontraron que los sujetos con TEA+TDAH obtuvieron peores puntuaciones en un inventario de calidad de vida relacionada con aspectos de salud que abarcaba diferentes áreas. Diversas investigaciones han informado sobre problemas de salud asociados al TEA en ámbitos como los problemas de sueño (Sivertsen, Posserud, Gillberg, Lundervold y Hysing, 2012) o problemas gastrointestinales (Mazurek et al., 2013). Estas complicaciones relacionadas con la salud pueden tener repercusiones importantes en la calidad de vida de quienes las padecen, por lo que la mayor presencia de problemas de calidad de vida relacionada con la salud en sujetos con diagnóstico de TEA+TDAH debe ser un aspecto importante a contemplar.

Consideramos que la presente revisión tiene repercusiones para la práctica clínica y educativa. Creemos que esta revisión puede ayudar a comprender la importancia de evaluar la presencia comórbida de TEA y TDAH, ya que se ha evidenciado que esta comorbilidad supone un aumento en la severidad de la sintomatología de TEA, así como otros trastornos internalizantes y externalizantes.

La presente investigación tiene diversas limitaciones como es el moderado número de trabajos incluidos en la misma. Debido a la reciente aceptación de la comorbilidad de estos dos trastornos, la comunidad científica todavía no ha llevado a cabo muchas investigaciones centradas específicamente en la relación entre el diagnóstico comórbido TEA + TDAH y los efectos que este tiene en la calidad de vida, el rendimiento académico o la calidad de vida familiar, lo cual ha dificultado la realización de la presente revisión. Otra de las limitaciones de nuestra investigación es que solo han sido revisadas algunas variables, por lo que en futuras investigaciones sería interesante llevar a cabo un estudio más profundo y amplio de otras variables como pueden ser el lenguaje y la comunicación o su relación con otras psicopatologías comórbidas como la ansiedad.

Pese a estas limitaciones, consideramos que el presente trabajo puede ser de ayuda para aquellos profesionales que deseen profundizar en el conocimiento de los efectos que provoca la presencia del trastorno comórbido TEA + TDAH, ya que aborda de forma sistemática los diferentes aspectos que se han estudiado hasta la fecha sobre esta comorbilidad. 


\section{Referencias}

American Psychiatric Association. (1994). Diagnostic and Statistical Manual of Mental Disorders (4a ed.) (DSM-IV). Washington DC (EEUU): APA.

American Psychiatric Association. (2013). Diagnostic and Statistical Manual of Mental Disorders (5a ed.) (DSM-V). Washington DC (EEUU): APA

Baio, J. (2012). Prevalence of Autism Spectrum Disorders: autism and developmental disabilities monitoring network, 14 Sites, United States, 2008. Morbidity and mortality weekly report. Surveillance Summaries. Centers for Disease Control and Prevention, 61(3), 1-19.

*Barnard-Brak, L., Sulak, T. N., y Fearon, D. D. (2011). Coexisting disorders and academic achievement among children with ADHD. Journal of Attention Disorders, 15(6), 506-515. doi: 10.1177/1087054710369667

Baron-Cohen, S., Scott, F. J., Allison, C., Williams, J., Bolton, P., Matthews, F. E., y Brayne, C. (2009). Prevalence of autism-spectrum conditions: UK school-based population study. The British Journal of Psychiatry, 194(6), 500-509. doi: 10.1192/bjp.bp.108.059345

Cardo, E., Servera, M., Vidal, C., De Azua, B., Redondo, M., y Riutort, L. (2011). Influencia de los diferentes criterios diagnósticos y la cultura en la prevalencia del trastorno por déficit de atención/hiperactividad. Revista de Neurología, 52(Supl 1), 109-17.

De la Iglesia Gutiérrez, M., y Olivar Parra, J. S. (2012). Revisión de estudios e investigaciones relacionadas con la comorbilidad diagnóstica de los Trastornos del Espectro del Autismo de Alto Funcionamiento (TEAAF) y los trastornos de ansiedad. Anales de Psicología, 28(3), 823-833. doi: 10.6018/analesps.28.3.124881

De Zwaan, M., Gruß, B., Müller, A., Graap, H., Martin, A., Glaesmer, H., .. y Philipsen, A. (2012). The estimated prevalence and correlates of adult ADHD in a German community sample. European Archives of Psychiatry and Clinical Neuroscience, 262(1), 79-86. doi: 10.1007/s00406-011-0211-9

Ekas, N., y Whitman, T. L. (2010). Autism symptom topography and maternal socioemotional functioning. Journal Information, 115(3), 234-249. doi: http://dx.doi.org/10.1352/1944-7558-115.3.234

Fombonne, E. (2003). Epidemiological surveys of autism and other pervasive developmental disorders: an update. Journal of Autism and Developmental Disorders, 33(4), 365-382. doi: 10.1023/A:1025054610557

Fombonne, E. (2005). The changing epidemiology of autism. Journal of Applied Research in Intellectual Disabilities, 18(4), 281-294. doi: 10.1111/j.14683148.2005.00266.x

Frazier, T. W., Youngstrom, E. A., Speer, L., Embacher, R., Law, P., Constantino, J., ... y Eng., C. (2012). Validation of proposed DSM-5 criteria for autism spectrum disorder. Journal of the American Academy of Child and Adolescent Psychiatry, 51(1), 28-40. doi: 10.1016/j.jaac.2011.09.021

*Gadow, K. D., DeVincent, C. J., y Pomeroy, J. (2006). ADHD symptom subtypes in children with pervasive developmental disorder. Journal of Autism and Developmental Disorders, 36(2), 271-283. doi: 10.1007/s10803005-0060-3

Gargaro, B. A., Rinehart, N. J., Bradshaw, J. L., Tonge, B. J., y Sheppard, D. M. (2011). Autism and ADHD: how far have we come in the comorbidity debate? Neuroscience \& Biobehavioral Reviews, 35(5), 1081-1088. doi: 10.1016/j.neubiorev.2010.11.002

Getahun, D., Jacobsen, S. J., Fassett, M. J., Chen, W., Demissie, K., y Rhoads, G. G. (2013). Recent trends in childhood attentiondeficit/hyperactivity disorder. JAMA pediatrics, 167(3), 282-288.doi: 10.1001/2013.jamapediatrics.401.

*Gjevik, E., Eldevik, S., Fjæran-Granum, T., y Sponheim, E. (2011). KiddieSADS reveals high rates of DSM-IV disorders in children and adolescents with autism spectrum disorders. Journal of Autism and Developmental Disorders, 41(6), 761-769. doi: 10.1007/s10803-010-1095-7

*Goldin, R. L., Matson, J. L., Tureck, K., Cervantes, P. E., y Jang, J. (2013). A comparison of tantrum behavior profiles in children with ASD, ADHD and comorbid ASD and ADHD. Research in Developmental Disabilities, 34(9), 2669-2675. doi: 10.1016/i.ridd.2013.04.022

*Goldstein, S., y Schwebach, A. J. (2004). The comorbidity of pervasive developmental disorder and attention deficit hyperactivity disorder: results of a retrospective chart review. Journal of Autism and Developmental Disorders, 34(3), 329-339. doi: 10.1023/B:JADD.0000029554.46570.68
*Grzadzinski, R., Di Martino, A., Brady, E., Mairena, M. A., O’Neale, M. Petkova, E., ... y Castellanos, F. X. (2011). Examining autistic traits in children with ADHD: does the autism spectrum extend to ADHD? Journal of Autism and Developmental Disorders, 41(9), 1178-1191. doi: 10.1007/s10803-010-1135-3

*Hanson, E., Cerban, B. M., Slater, C. M., Caccamo, L. M., Bacic, J., y Chan, E. (2013). Brief report: Prevalence of attention deficit/hyperactivity disorder among individuals with an autism spectrum disorder. Journal of Autism and Developmental Disorders, 43(6), 1459-1464. doi: $10.1007 / \mathrm{s} 10803-012-1677-7$

Hayes, S. A., y Watson, S. L. (2013). The impact of parenting stress: A metaanalysis of studies comparing the experience of parenting stress in parents of children with and without autism spectrum disorder. Journal of Autism and Developmental Disorders, 43(3), 629-642. doi: 10.1007/s10803$012-1604-y$

*Holtmann, M., Bölte, S., y Poustka, F. (2007). Attention deficit hyperactivity disorder symptoms in pervasive developmental disorders: association with autistic behavior domains and coexisting psychopathology. Psychopathology, 40(3), 172-177. doi: 10.1159/000100007

Hsu, S. W., Chiang, P. H., Lin, L. P., y Lin, J. D. (2012). Disparity in autism spectrum disorder prevalence among Taiwan National Health Insurance enrollees: Age, gender and urbanization effects. Research in Autism Spectrum Disorders, 6(2), 836-841. doi: 10.1016/j.rasd.2011.09.006

Huerta, M., Bishop, S. L., Duncan, A., Hus, V., y Lord, C. (2012). Application of DSM-5 criteria for autism spectrum disorder to three samples of children with DSM-IV diagnoses of pervasive developmental disorders. American Journal of Psychiatry, 169(10), 1056-1064. doi: 10.1176/appi.ajp.2012.12020276

*Jang, J., Matson, J. L., Cervantes, P. E., y Goldin, R. L. (2013b). The relationship between race and comorbid symptoms in infants and toddlers with autism spectrum disorder. Research in Autism Spectrum Disorders, 7(11), 1433-1438. doi: 10.1016/j.rasd.2013.08.011

*Jang, J., Matson, J. L., Williams, L. W., Tureck, K., Goldin, R. L., y Cervantes, P. E. (2013a). Rates of comorbid symptoms in children with ASD, ADHD, and comorbid ASD and ADHD. Research in Developmental Disabilities, 34(8), 2369-2378. doi: 10.1016/j.ridd.2013.04.021

Kaland, N. (2011). Brief report: should Asperger syndrome be excluded from the forthcoming DSM-V? Research in Autism Spectrum Disor ders, 5(3), 984-989. doi: 10.1016/j.rasd.2011.01.011

*Konst, M. J., Matson, J. L., y Turygin, N. (2013). Comparing the rates of tantrum behavior in children with ASD and ADHD as well as children with comorbid ASD and ADHD diagnoses. Research in Autism Spectrum Disorders, 7(11), 1339-1345. doi: 10.1016/j.rasd.2013.07.023

*Kotte, A., Joshi, G., Fried, R., Uchida, M., Spencer, A., Woodworth, K. Y. ... \& Biederman, J. (2013). Autistic traits in children with and without ADHD. Pediatrics, 132(3), e612-e622. doi: 10.1542/peds.2012-3947

*Lee, D. O., y Ousley, O. Y. (2006). Attention-deficit hyperactivity disorder symptoms in a clinic sample of children and adolescents with pervasive developmental disorders. Journal of Child \& Adolescent Psychopharmacology, 16(6), 737-746. doi: 10.1089/cap.2006.16.737

Lin, J. D., Sung, C. L., Lin, L. P., Hsu, S. W., Chien, W. C., Su, S. L., y Wu, J. L. (2011). Ten-year trend analysis of autism severity: A nationwide population-based register study. Research in Autism Spectrum Disorders, 5(1), 570-574. doi: 10.1016/j.rasd.2010.06.024

Lyons, A. M., Leon, S. C., Phelps, C. E. R., y Dunleavy, A. M. (2010). The impact of child symptom severity on stress among parents of children with ASD: The moderating role of coping styles. Journal of Child and Family Studies, 19(4), 516-524 doi: 10.1007/s10826-009-9323-5

*Mannion, A., Leader, G., y Healy, O. (2013). An investigation of comorbid psychological disorders, sleep problems, gastrointestinal symptoms and epilepsy in children and adolescents with autism spectrum disorder. Research in Autism Spectrum Disorders, 7(1), 35-42. doi: 10.1016/j.rasd.2012.05.002

Martín-Fernández, J. D. (2013). La (no) decepción del DSM-5. Cuadernos de Neuropsicología, 7(1), 9-21. 
Matson, J. L., y Kozlowski, A. M. (2011). The increasing prevalence of autism spectrum disorders. Research in Autism Spectrum Disorders, 5(1), 418425. doi: 10.1016/j.rasd.2010.06.004

*May, T., Rinehart, N., Wilding, J., y Cornish, K. (2013). The role of attention in the academic attainment of children with autism spectrum disorder. Journal of Autism and Developmental Disorders, 43(9), 2147-2158. doi: 10.1007/s10803-013-1766-2

Mazurek, M. O., Vasa, R. A., Kalb, L. G., Kanne, S. M., Rosenberg, D., Keefer, A., ... y Lowery, L. A. (2013). Anxiety, sensory overresponsivity, and gastrointestinal problems in children with autism spectrum disorders. Journal of Abnormal Child Psychology, 41(1), 165-176. doi: 10.1007/s10802-012-9668-x

*Miranda, A., Tárraga, R., Fernández, M. I., Colomer, C., y Pastor, G. (2015). Parenting Stress in Families of Children with Autism Spectrum Disorder and ADHD. Exceptional Children, preprint.doi: 10.1177/00144029155854790014402915585479.

Polanczyk, G., de Lima, M. S., Horta, B. L., Biederman, J., y Rohde, L. A. (2007). The worldwide prevalence of ADHD: a systematic review and metaregression analysis. The American journal of psychiatry, 164(6), 942948. doi: 10.1176/ajp.2007.164.6.942

Pozo, P., y Sarriá, E. (2014). Un modelo global de estrés en padres de personas con trastornos del espectro autista. Anales de Psicología, 30(1), 180191. doi: 10.618 /analesps.30.1.140722

*Rao, P. A., y Landa, R. J. (2014). Association between severity of behavioral phenotype and comorbid attention deficit hyperactivity disorder symptoms in children with autism spectrum disorders. Autism, 18(3), 272280. doi: $10.1177 / 1362361312470494$

*Reiersen, A. M., Constantino, J. N., Volk, H. E., y Todd, R. D. (2007). Autistic traits in a population-based ADHD twin sample. Journal of Child Psychology and Psychiatry, 48(5), 464-472. doi: 10.1111/j.14697610.2006.01720.x

Ritvo, E. R. (2012). Postponing the proposed changes in DSM 5 for autistic spectrum disorder until new scientific evidence adequately supports them. Journal of Autism and Developmental Disorders, 42(9), 1-2. doi: 10.1007/s10803-012-1613-x

*Russell, G., Rodgers, L. R., Ukoumunne, O. C., y Ford, T. (2014). Prevalence of parent-reported ASD and ADHD in the UK: findings from the Millennium Cohort Study. Journal of Autism and Developmental Disorders, 44(1), 31-40. doi: 10.1007/s10803-013-1849-0

*Sikora, D. M., Vora, P., Coury, D. L., y Rosenberg, D. (2012). Attentiondeficit/hyperactivity disorder symptoms, adaptive functioning, and quality of life in children with autism spectrum disorder. Pediatrics, 130(2), 91-97. doi: 10.1542/peds.2012-0900G

Simon, V., Czobor, P., y Bitter, I. (2013). Is ADHD severity in adults associated with the lifetime prevalence of comorbid depressive episodes and anxiety disorders? European Psychiatry, 28(5), 308-314. doi: 10.1016/j.eurpsy.2012.05.002
*Simonoff, E., Pickles, A., Charman, T., Chandler, S., Loucas, T., y Baird, G. (2008). Psychiatric disorders in children with autism spectrum disorders: prevalence, comorbidity, and associated factors in a population-derived sample. Journal of the American Academy of Child \& Adolescent Psychiatry, 47(8), 921-929. doi: 10.1097/CHI.0b013e318179964f

*Sinzig, J., Walter, D., y Doepfner, M. (2009). Attention Deficit/Hyperactivity Disorder in children and adolescents with Autism Spectrum Disorder symptom or syndrome? Journal of Attention Disorders, 13(2), 117-126. doi: 10.1177/1087054708326261

Sivertsen, B., Posserud, M. B., Gillberg, C., Lundervold, A. J., y Hysing, M. (2012). Sleep problems in children with autism spectrum problems: a longitudinal population-based study. Autism, 16(2), 139-150. doi: $10.1177 / 1362361311404255$

*Sprenger, L., Bühler, E., Poustka, L., Bach, C., Heinzel-Gutenbrunner, M., Kamp-Becker, I., y Bachmann, C. (2013). Impact of ADHD symptoms on autism spectrum disorder symptom severity. Research in Developmental Disabilities, 34(10), 3545-3552. doi: 10.1016/j.ridd.2013.07.028

Theule, J., Wiener, J., Tannock, R., y Jenkins, J. M. (2013). Parenting stress in families of children with ADHD. A Meta-Analysis. Journal of Emotional and Behavioral Disorders, 21(1), 3-17. doi: 10.1177/1063426610387433

*Tureck, K., Matson, J. L., May, A., y Turygin, N. (2013). Externalizing and tantrum behaviours in children with ASD and ADHD compared to children with ADHD. Developmental Neurorehabilitation, 16(1), 52-57. doi: 10.3109/17518423.2012.719245

*Turygin, N., Matson, J. L., y Tureck, K. (2013). ADHD symptom prevalence and risk factors in a sample of toddlers with ASD or who are at risk for developmental delay. Research in developmental disabilities, 34(11), 4203-4209. doi:10.1016/j.ridd.2013.07.020

*Van Steijn, D. J., Oerlemans, A. M., van Aken, M. A., Buitelaar, J. K., y Rommelse, N. N. (2013). Match or mismatch? Influence of parental and offspring ASD and ADHD symptoms on the parent-child relationship. Journal of Autism and Developmental Disorders, 43(8), 1935-1945. doi: 10.1007/s10803-012-1746-y

*Van Steijn, D. J., Oerlemans, A. M., van Aken, M. A., Buitelaar, J. K., y Rommelse, N. N. (2014). The reciprocal relationship of ASD, ADHD, depressive symptoms and stress in parents of children with ASD and/or ADHD. Journal of Autism and Developmental Disorders, 44(5), 1-13. doi: 10.1007/s10803-013-1958-9

Wing, L., Gould, J., y Gillberg, C. (2011). Autism spectrum disorders in the DSM-V: better or worse than the DSM-IV? Research in Developmental Disabilities, 32(2), 768-773. doi: 10.1016/j.ridd.2010.11.003

*Yerys, B. E., Wallace, G. L., Sokoloff, J. L., Shook, D. A., James, J. D., y Kenworthy, L. (2009). Attention deficit/hyperactivity disorder symptoms moderate cognition and behavior in children with autism spectrum disorders. Autism Research, 2(6), 322-333. doi: 10.1002/aur.103

(Artículo recibido: 13-01-2015; revisado: 05-10-2015; aceptado: 18-11-2015) 